NASA/TM-2004-213074

Evolutionary Space Communications Architectures for Human/Robotic Exploration and Science Missions

Kul Bhasin

Glenn Research Center, Cleveland, Ohio

Jeffrey L. Hayden

PresciPoint Solutions, L.L.C., Littleton, Colorado 
Since its founding, NASA has been dedicated to the advancement of aeronautics and space science. The NASA Scientific and Technical Information (STI) Program Office plays a key part in helping NASA maintain this important role.

The NASA STI Program Office is operated by Langley Research Center, the Lead Center for NASA's scientific and technical information. The NASA STI Program Office provides access to the NASA STI Database, the largest collection of aeronautical and space science STI in the world. The Program Office is also NASA's institutional mechanism for disseminating the results of its research and development activities. These results are published by NASA in the NASA STI Report Series, which includes the following report types:

- $\quad$ TECHNICAL PUBLICATION. Reports of completed research or a major significant phase of research that present the results of NASA programs and include extensive data or theoretical analysis. Includes compilations of significant scientific and technical data and information deemed to be of continuing reference value. NASA's counterpart of peerreviewed formal professional papers but has less stringent limitations on manuscript length and extent of graphic presentations.

- TECHNICAL MEMORANDUM. Scientific and technical findings that are preliminary or of specialized interest, e.g., quick release reports, working papers, and bibliographies that contain minimal annotation. Does not contain extensive analysis.

- CONTRACTOR REPORT. Scientific and technical findings by NASA-sponsored contractors and grantees.
- CONFERENCE PUBLICATION. Collected papers from scientific and technical conferences, symposia, seminars, or other meetings sponsored or cosponsored by NASA.

- SPECIAL PUBLICATION. Scientific, technical, or historical information from NASA programs, projects, and missions, often concerned with subjects having substantial public interest.

- TECHNICAL TRANSLATION. Englishlanguage translations of foreign scientific and technical material pertinent to NASA's mission.

Specialized services that complement the STI Program Office's diverse offerings include creating custom thesauri, building customized databases, organizing and publishing research results ... even providing videos.

For more information about the NASA STI Program Office, see the following:

- Access the NASA STI Program Home Page at http://www.sti.nasa.gov

- E-mail your question via the Internet to help@sti.nasa.gov

- Fax your question to the NASA Access Help Desk at 301-621-0134

- Telephone the NASA Access Help Desk at 301-621-0390

- Write to:

NASA Access Help Desk

NASA Center for AeroSpace Information 7121 Standard Drive

Hanover, MD 21076 
NASA/TM-2004-213074

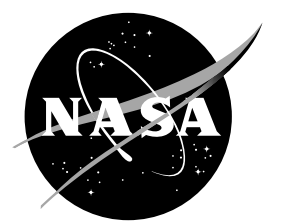

\section{Evolutionary Space Communications Architectures for Human/Robotic Exploration and Science Missions}

Kul Bhasin

Glenn Research Center, Cleveland, Ohio

Jeffrey L. Hayden

PresciPoint Solutions, L.L.C., Littleton, Colorado

Prepared for the

Space Technology and Applications International Forum (STAIF-2004) sponsored by the American Institute of Physics Albuquerque, New Mexico, February 8-12, 2004

National Aeronautics and Space Administration

Glenn Research Center 


\section{Acknowledgments}

The authors would like to thank O.S. Sands for his assistance in accumulating the Moon vicinity information, and K. Perko, for his assistance in obtaining data on possible TDRSS improvements.

This report contains preliminary

findings, subject to revision as analysis proceeds.

Available from

NASA Center for Aerospace Information 7121 Standard Drive

Hanover, MD 21076
National Technical Information Service 5285 Port Royal Road Springfield, VA 22100 


\title{
Evolutionary Space Communications Architectures for Human/Robotic Exploration and Science Missions
}

\author{
Kul Bhasin \\ National Aeronautics and Space Administration \\ Glenn Research Center \\ Cleveland, Ohio 44135 \\ Jeffrey L. Hayden \\ PresciPoint Solutions, L.L.C. \\ Littleton, Colorado 80123
}

\begin{abstract}
NASA enterprises have growing needs for an advanced, integrated, communications infrastructure that will satisfy the capabilities needed for multiple human, robotic and scientific missions beyond 2015. Furthermore, the reliable, multipoint infrastructure is required to provide continuous, maximum coverage of areas of concentrated activities, such as around Earth and in the vicinity of the Moon or Mars, with access made available on demand of the human or robotic user. As a first step, the definitions of NASA's future space communications and networking architectures are underway. Architectures that describe the communications and networking needed between the nodal regions consisting of Earth, Moon, Lagrange points, Mars, and the places of interest within the inner and outer solar system have been laid out. These architectures will need the modular flexibility that must be included in the communication and networking technologies to enable the infrastructure to grow in capability with time and to transform from supporting robotic missions in the solar system to supporting human ventures to Mars, Jupiter, Jupiter's moons, and beyond. The protocol-based networking capability seamlessly connects the backbone, access, inter-spacecraft and proximity network elements of the architectures employed in the infrastructure. In this paper, we present the summary of NASA's near and long term needs and capability requirements that were gathered by participative methods. We describe an integrated architecture concept and model that will enable communications for evolutionary robotic and human science missions. We then define the communication nodes, their requirements, and various options to connect them.
\end{abstract}

\section{Introduction}

Space communications architectures and technologies in the $21^{\text {st }}$ century must meet the growing needs of Earth sensor web and collaborative observation formation missions, robotic scientific missions for detailed investigation of planets, moons, and small bodies in the solar system, human missions for exploration of the Moon, Mars, Ganymede, Callisto, and asteroids, human settlements in space, on the Moon, and on Mars, and great in-space observatories for observing other star systems and the universe. An advanced, integrated, communications infrastructure will enable the reliable, multipoint, high data rate capabilities needed on demand to provide continuous, maximum coverage of areas of concentrated activities, such as in the vicinity of in-space outposts, the Moon or Mars.

Past work in space communications was developed from the several unrelated perspectives of the different enterprises with a view toward providing communication services for each of their new mission as they came along. Communications for Earth observing missions, for instance, were developed independently from what was needed for other missions such as the human shuttle and ISS missions. Communications for Mars and deep space missions also developed independently from the others and shared the use of the Deep Space Network (DSN). Communications were again treated from a services 
perspective and while the interfaces and protocols used for different missions were standardized, the standards could not support autonomous networking and data routing. More recently, the enterprises have been accumulating the capabilities that are felt to be necessary for future missions. However, the enterprise solutions identified for future communications remain services-centric, that is the solutions are specific to each enterprise's missions and are not integrated into an overall NASA communication infrastructure solution wherein the in-space nodes can communicate with each other as well as with users on Earth through the Internet. The commercial Iridium communication satellite constellation, while not as successful as originally anticipated, did prove that inter-spacecraft communications and networking was possible.

The approach taken in this paper is architecture-centric in that the work will lead to an integrated, inter-networked, space communications infrastructure developed from architectural elements and interfaces. Within this networked infrastructure, data will move from sensor to user under autonomous control of the nodes within the network. Human operations will become maintenance and network administrative functions. To obtain the requirements that follow, node-to-node link capability needs were captured from data provided by the enterprise mission planners and technologists. These capabilities include data rates, distance, and function needed over each general link from the Earth-side network and terminal to the in-space user node. Later work will extend into defining and standardizing hardware and software interfaces to be implemented in each node and identifying the most appropriate technologies to implement for those nodes. It is expected this architectural development work will need to continue as the infrastructure is first emplaced and then as it grows with time.

In this paper, we describe an integrated communications architecture that will support NASA's future human and robotic missions; we provide a summary of the communications needs and capabilities that the nodes in the resulting new infrastructure will satisfy; we then identify the architectural tradeoffs and the technology gaps that must be resolved to achieve a workable new architecture.

\section{Architecture Needs and Requirements}

The high level mission communication data rate requirements in Table 1 and required characteristics that follow motivate the need for a set of links between nodes of NASA's future space architecture. These capabilities are addressed by examining individual node-to-node links. The resulting architecture is then to be used to identify and focus technology development needed to support the physical network of communications links. Once the new technologies are in place in the physical architecture, the required high-level capabilities will be fully realized.

TABLE 1. Infrastructure Requirements.

\begin{tabular}{llccc}
\hline Nodal Group & Node to Earth & Current & $\mathbf{2 0 1 0}$ & $\mathbf{2 0 2 0 +}$ \\
\hline Earth Vicinity & LEO Spacecraft (Direct Link) & $150 \mathrm{Mbps}$ & $>1$ Gbps gateway, 1 Gbps D/L & $10 \mathrm{Gbps}$ \\
& GEO Spacecraft (Direct Link) & $150 \mathrm{Mbps}$ & $>1 \mathrm{Gbps}$ & $10 \mathrm{Gbps}$ \\
& STS & $50 \mathrm{Mbps}$ & $50 \mathrm{Mbps}$ & $50 \mathrm{Mbps}$ \\
\multirow{4}{*}{ Moon } & ISS & $48 \mathrm{Mbps}$ & $150 \mathrm{Mbps}(2005)$ & $300 \mathrm{Mbps}$ \\
& Earth-Moon L1, L2 & & & $0.2 \mathrm{up} / 1 \mathrm{down} \mathrm{Gbps}$ \\
Earth-Sun L1, L2 & GEO relay and Earth & & $20 \mathrm{Mbps}$ & $0.2 \mathrm{up} / 1 \mathrm{down} \mathrm{Gbps}$ \\
Mars & Mars Science & $100 \mathrm{Kbps}$ & $5 \mathrm{Mbps}$ & $>100 \mathrm{Mbps}$ \\
& Mars Exploration & - & $10 \mathrm{Mbps}$ & 20 up/100 down Mbps \\
& Mars Proximity Link & - & - & 20 up/100 down Mbps \\
Outer Planets & Jupiter to Outer Heliosphere & $10 \mathrm{Kbps}$ & $1 \mathrm{Mbps}$ & $1-100 \mathrm{Mbps}$ \\
\hline
\end{tabular}

The infrastructure will grow in an integrated fashion and evolve to support the missions of the future, rather than change in the independent, mission-specific way that it grew to support the exploratory missions of the past. The characteristics required by the evolving infrastructure are shown in Table 2 . 


\section{Evolutionary Space Communications Architecture Model}

NASA's communication infrastructure will become an autonomously operated system of networks on the ground and in-space. It will be possible for an in-space human or robotic spacecraft, rover, or groundbased user to demand and receive access to an arm of the network from nearly anywhere on or around the Earth, the Moon, or the Solar System. An integrated architectural scenario that implements an infrastructure with the desired characteristics is made up of several regions of interest where groups of communication nodes represented by science and human missions are likely to need access to modern networked, high data rate communications for conveying images, science data, voice, video, and control data between themselves and with Earth. The nodal regions of interest include the Earth vicinity from its surface to high Earth orbits, the Moon vicinity from lunar surface to the near and far Earth-Moon Lagrangian halo orbits $\left(\mathrm{EM}_{\mathrm{L} 1}\right.$ and $\left.\mathrm{EM}_{\mathrm{L} 2}\right)$, the halo orbits in the Earth-Sun Lagrangian vicinities $\left(\mathrm{ES}_{\mathrm{L} 1}\right.$, $\mathrm{ES}_{\mathrm{L} 2}, \mathrm{ES}_{\mathrm{L} 4}, \mathrm{ES}_{\mathrm{L} 5}$ ), Mars vicinity from its surface to the Mars synchronous orbit, Jupiter vicinity from its atmosphere to its Jupiter-Sun Lagrangian orbits $\left(\mathrm{JS}_{\mathrm{L} 1}, \mathrm{JS}_{\mathrm{L} 2}\right)$, the neighborhoods of the rest of the planets, moons and objects in the Solar System. The architectural scenario described below implements the evolutionary space communications architecture, its architectural elements and interfaces, the science it supports, and its concept of operations.

TABLE 2. Required Characteristics of the Infrastructure.

\begin{tabular}{|c|c|}
\hline Required Characteristic & Rationale \\
\hline Be available 24/7. & Basic requirement of human missions and most missions requiring low latency data return. \\
\hline Integrated Architectures & $\begin{array}{l}\text { Use of standard interfaces (hardware, wireless, and protocols) across the infrastructure } \\
\text { increases data routing options and reduces costs of implementation. }\end{array}$ \\
\hline Low cost, modular and efficient. & This can be achieved by adapting of commercial technology standards to use in space. \\
\hline $\begin{array}{l}\text { Handle multipoint connections to } \\
\text { multiple nodes simultaneously. }\end{array}$ & $\begin{array}{l}\text { Essential for broadcasting data to many spacecraft simultaneously; for inter-spacecraft } \\
\text { coordination of timing, maneuvers, and collaborative science data gathering; and for } \\
\text { enabling autonomous end-to-end routing of data. }\end{array}$ \\
\hline Highly reliable connections & $\begin{array}{l}\text { Connections must be reliable to meet the very high data rates, else the required } \\
\text { characteristics will not be met. }\end{array}$ \\
\hline Long life expectancy. & High cost of development and space flight dictates lifetimes of greater than 20 years. \\
\hline Highly reconfigurable & To accommodate upgrades and enable growth in capabilities over time. \\
\hline Be secure. & Cannot allow intruders to take control of the systems nor allow sampling of private data. \\
\hline Connect End-to-end & $\begin{array}{l}\text { Enabling data to move on demand from user to spacecraft instrument or back greatly } \\
\text { reduces operations support costs. }\end{array}$ \\
\hline $\begin{array}{l}\text { Handle multiple robotic and } \\
\text { human missions simultaneously. }\end{array}$ & $\begin{array}{l}\text { Essential for providing communication routes for many spacecraft simultaneously so that } \\
\text { many data streams can be routed from end-to-end autonomously. }\end{array}$ \\
\hline Multiple quality of service levels & $\begin{array}{l}\text { QoS diversity is required to handle voice, video, science data and control data } \\
\text { simultaneously. }\end{array}$ \\
\hline $\begin{array}{l}\text { Minimum latency within the } \\
\text { networks. }\end{array}$ & $\begin{array}{l}\text { Required for maintaining the tightest possible control loops that are necessary in most } \\
\text { human-operated remote missions. It also helps for keeping human-human communications } \\
\text { as close to real-time as possible. }\end{array}$ \\
\hline $\begin{array}{l}\text { Provide navigation capabilities } \\
\text { within telemetry signals. }\end{array}$ & Needed for missions that must coordinate their activities and for flying in formations. \\
\hline Operate in extreme environments & $\begin{array}{l}\text { In-space hardware must survive solar flares and cold temperatures. Planetary/moon } \\
\text { hardware faces large temperature swings (Moon, Mars), high radiation (Europa), high } \\
\text { temperature (Mercury). }\end{array}$ \\
\hline
\end{tabular}

\section{Architecture Scenario-Description}

The proposed integrated architecture scenario is illustrated in Figure 1. This scenario was developed based on NASA's needs and requirements collected through participative processes. This is a first attempt to look at the space communication architecture in an integrated fashion while addressing the needs of the NASA enterprises. The figure shows the scenario of a networked space communications infrastructure 
with connections to the regions of interest within the solar system. The communication capabilities are provided by a constellation of geosynchronous Earth orbiting (GEO) communications relay satellites, sensor web inter-spacecraft communications packages for relaying data between science observation satellites, high data rate, small, autonomous ground terminals, communications relay spacecraft placed in gravitationally balanced Lagrange orbits between the Earth and Moon, the Earth and sun, and Jupiter and the sun, relay satellites around the Moon, and science and relay satellites placed in orbit around Mars, the outer planets and small bodies. The communication links shown in the figure are further described below.

Architecture elements and interfaces.-The integrated communications architecture diagramed in Figure 1 can be represented by four architectural elements (Bhasin, 2002): High Rate Backbone Elements - whose inter-nodal links are represented by double light grey lines; Access Network Elements - these links are shown as single medium grey lines; Inter-Spacecraft Elements - black lines with diamond shaped arrow heads; and Proximity Elements - black dotted lines. Collectively, links within and between these elements represent segments of the pathways needed to achieve the end-to-end data-passing capability envisioned for future NASA communications. The high rate backbone network elements are the intra-network structures of high rate communication nodes and inter-nodal links that utilize advanced communication technologies to increase data rate by orders of magnitude while reducing overall costs. The flexible access network elements are re-configurable communication systems at the edges of the backbone networks that enable in-space humans, robotic spacecraft, aircraft, or ground vehicles to communicate to the infrastructure edge-nodes. Inter-spacecraft cooperative network elements incorporate the technologies necessary to enable intercommunications between future NASA spacecraft flying in formation, in clusters, or in constellations. Proximity wireless network elements include: short range, low power, low cost, short-lived communications packages for inter-communication between small sensor packages; and small wireless local area network (WLAN) packages to support high data rate, bidirectional communications for voice, video, data, and control between humans and robots over a distance of meters to a few kilometers.

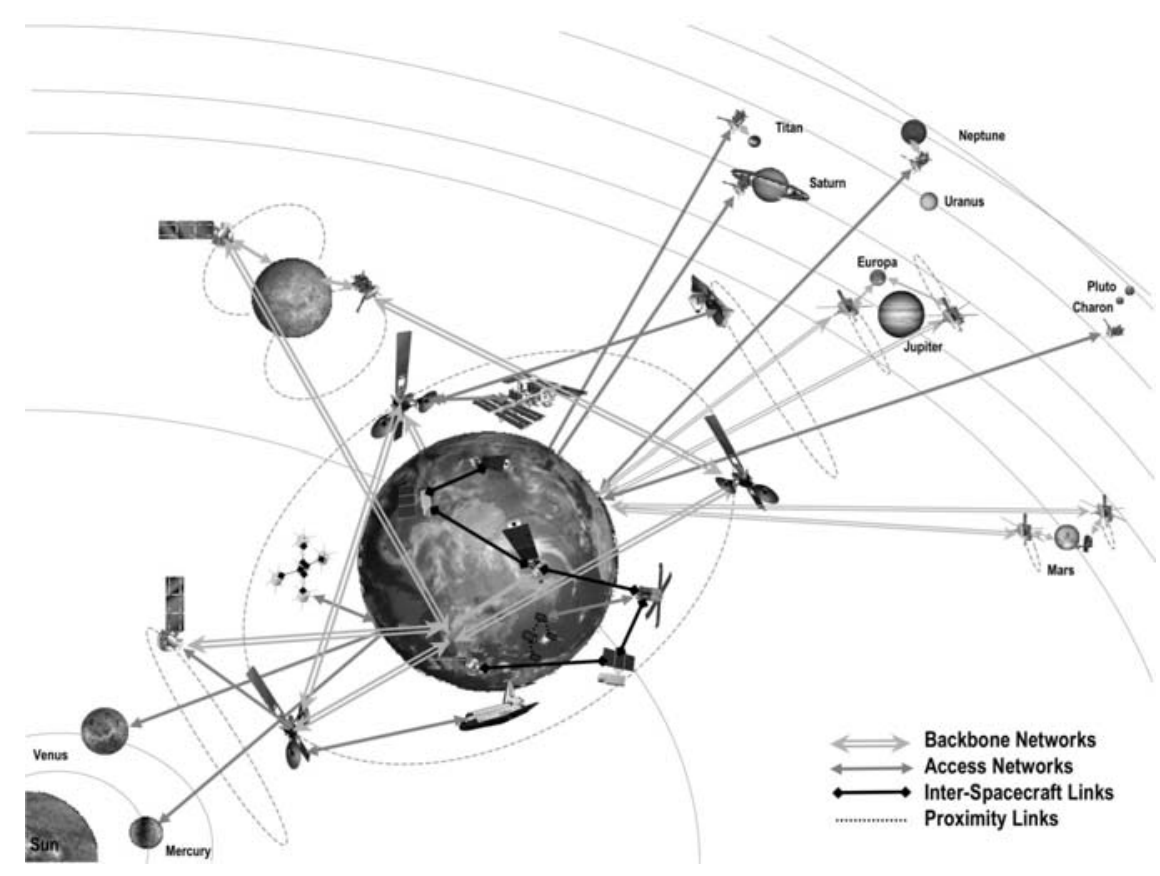

FIGURE 1. Integrated Architecture Scenario. 
Science supported by the space communication architectures.-NASA's communications infrastructure must support all varieties of science and human exploration in the future. The science to be supported ranges from observation of the Earth, moon, Mars, the outer planet systems, to the universe. The science also includes that which is obtained during human exploration and inhabitation of space, the moon, Mars, and outer planet moons. Most of the NASA science missions that are under study require high-bandwidth communications, including (in very short summary): hyperspectral imagery, synthetic aperture radar imagery, atmospheric measurements, and radar sounding of planetary/moon bodies; astronomical imagery from radio frequencies to gamma rays of other star systems, the galaxy, and universe; robotic measurements of planet/moon surface and atmospheric properties; and the search for life by many means.

Operations concept.-This NASA infrastructure is a key part of the operations concepts for all future missions. NASA's missions will evolve to be more self-supporting. Many robotic missions will operate autonomously by sensing the area around them and making decisions about where to go, what samples to measure, what data to report, and for requesting and connecting to the space communication network. Other robotic entities will be intimately connected to human operators via wireless systems that enable real-time, or delayed-time video and control for close coordination such as in assembling large space structures. The goal of the infrastructure design is to become a space Internet that is as autonomous as possible in operation and where connections are made and broken as needed by the requesting entity. This kind of communications infrastructure will allow access on the demand of any mission entity, including spacecraft, surface robot, in-space exploring human, and Earth user, while using as few human operators as possible to provide the capabilities. The communications relay concept shown in Figure 2 is posed as a series of high data rate communication relay spacecraft that can act as deep space network nodes capable of making multiple simultaneous connections to missions scattered around the solar system. A network of nodes made of these clustered and tethered communication components can support the autonomous routing of data streams between any two nodes in the solar system and also provides alternate communications paths around the sun when a target node is in conjunction with the sun.

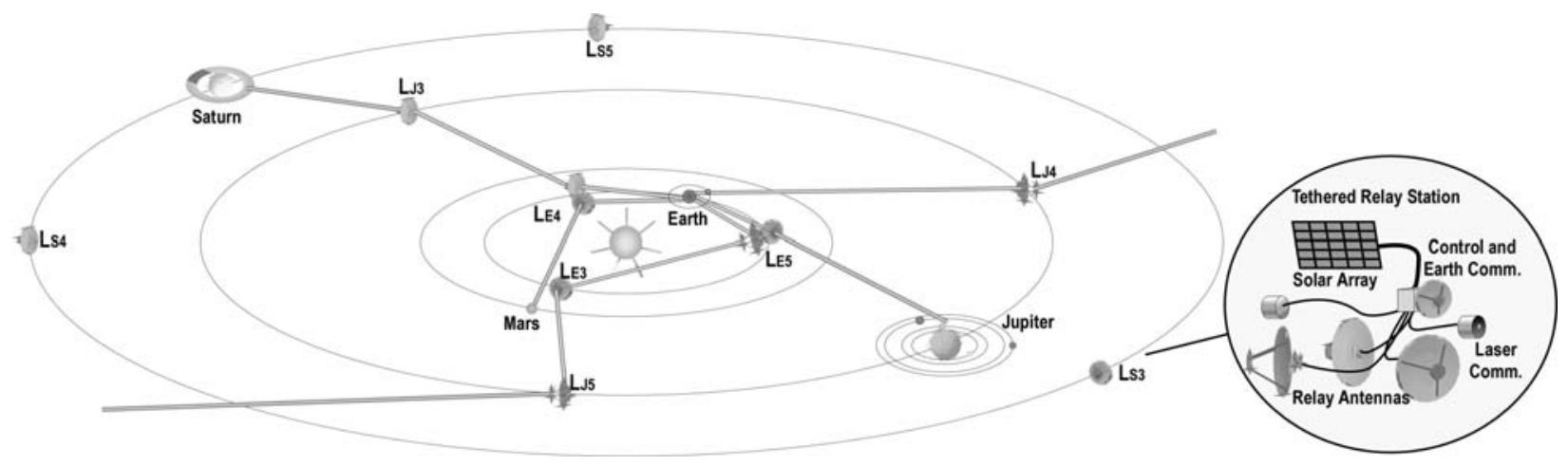

FIGURE 2. A Communications Relay Constellation for Providing Networked Links to Mars and the Outer Planets.

Implementation technologies.-The technologies that will be used in the implementation the architectures include, but are not limited to: microwave antennas, receivers, transmitters, and modulators; optical telescopes, receivers, lasers, and modulators; in-space networking routers, circuit switches, and network interface modules; and networking protocols and autonomous applications. Communication characteristics are identified for the link between each node pair that is expected to intercommunicate. Technologies are then chosen that have the best chance of providing those characteristics. The driving capabilities of very high data rates and inter-networking that must be met lead to new developments in microwave and optical communications components and systems, in-space networking systems, and network protocol and application software. 


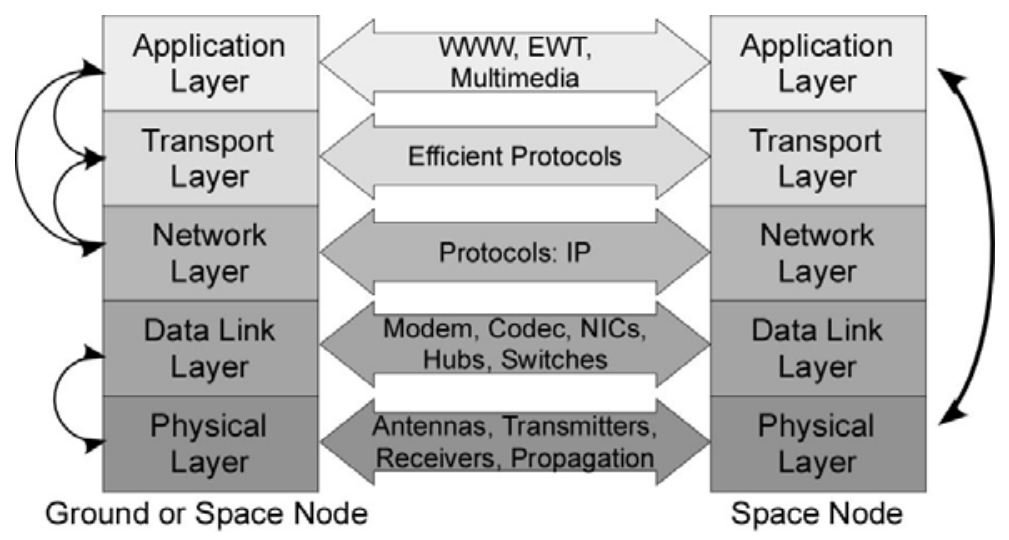

FIGURE 3. Internet Protocol Layers used in Integrated Architecture.

\section{Layered/Integrated Communications Architecture}

With integrated architectures, NASA will be able to achieve intelligent communications. The communication networking paths will utilize the lower five of the seven Open System Interconnection (OSI) model layers (Figure 3), to achieve Internet-like data routing capabilities. Current approaches have only nominal interaction between these layers (Shames, 2003). However, interactive control between the layers enables autonomous data routing on-board and between spacecraft by allowing control of antenna pointing, transmitter power, transmit data rates and media access methods that vary with distance, thus permitting a complete end-to-end data routing capability. It also enables spacecraft or users to demand access to the network as if it were making a cellular phone call. Common protocols and interfaces at these layers will enable inter-active links to be made and broken on demand of any node in the network, thus enabling complex and deeply networked communications channels between nodes in space and on Earth.

\section{Communication Nodes-Descriptions and Options}

As the next step, the individual communication nodes within each region of the evolutionary architecture model were identified. These nodes included all entities (sensors, spacecraft, aircraft, robots, humans, etc.) that might communicate with each other inside or outside of the region. Then links for each pair of nodes that might reasonably be expected to inter-communicate were identified. This provided a view of all the links into and out of a particular node and a means to tabulate their physical and desired characteristics. These node-to-node links become the optional building blocks of the architectures. There are multiple paths by which data can move from one node to another. The existence of a path depends on whether a particular architectural element option is chosen for implementation into the infrastructure. Many node-to-node link options will likely drop out of consideration with further analysis.

The Earth vicinity communications nodal group encompasses the communications infrastructure needed to support robotic and human missions from the Earth surface to high Earth orbit (HEO). It includes: that part of the DoD's transformational communications architecture (TCA) (Armstrong, 2003) that NASA may implement and/or use; communication relay satellite networks that may optionally be placed in geosynchronous Earth orbits (GEO), or high inclination Molniya orbits, medium Earth orbit (MEO), and low Earth orbit (LEO) Earth observer satellite data and command paths. The Moon vicinity nodal group encompasses the surface and orbits of the Moon and the Earth-Moon system's Lagrange points. Elements of the physical communications infrastructure considered in this group include: communication relay satellites in Earth-Moon Lagrange orbit, or Moon orbit, long-link Moon to Earth communications, and wireless local area networks (WLANs) on the surface of the Moon. The Earth-Sun 
Lagrangian vicinity nodal group comprises those elements of the communications infrastructure that might be placed at the Earth-Sun Lagrange points L1, L2, L4, and/or L5 to provide high data rate backbone capabilities for Earth, sun, galaxy, or universe observing missions and deep space science missions. The Mars vicinity nodal group encompasses communications infrastructure that might be implemented to support robotic and human missions at Mars. It includes: a relay satellite network for Mars that might optionally be placed in Mars synchronous orbit (MSO), Mars high orbit (MHO), and/or Mars low orbit (MLO); networks for Mars orbit, air, and surface robotic missions; and Mars human outpost communication networks. The deep space communications nodal group is the communications infrastructure that is dispersed among the outer planets and moons in support of robotic and later human missions. It includes outer planet mission communication systems and communication relay spacecraft that might be placed in Jupiter-Sun L1, L2 halo orbits.

\section{Earth Vicinity Communication Nodes}

The Earth vicinity communications infrastructure for observation and exploration missions is diagrammed in Figure 4 and includes the LEO, MEO, GEO, HEO relay satellites that may be implemented.

The architectural elements and their options for the Earth vicinity communications infrastructure are listed below. Within each of the options, multiple node-to-node link possibilities have been identified and characterized. A listing of all of the options' node-to-node links is very extensive and beyond the scope of this paper. Consequently, an example of the characterization of one of the option's set of node-to-node links is given below.

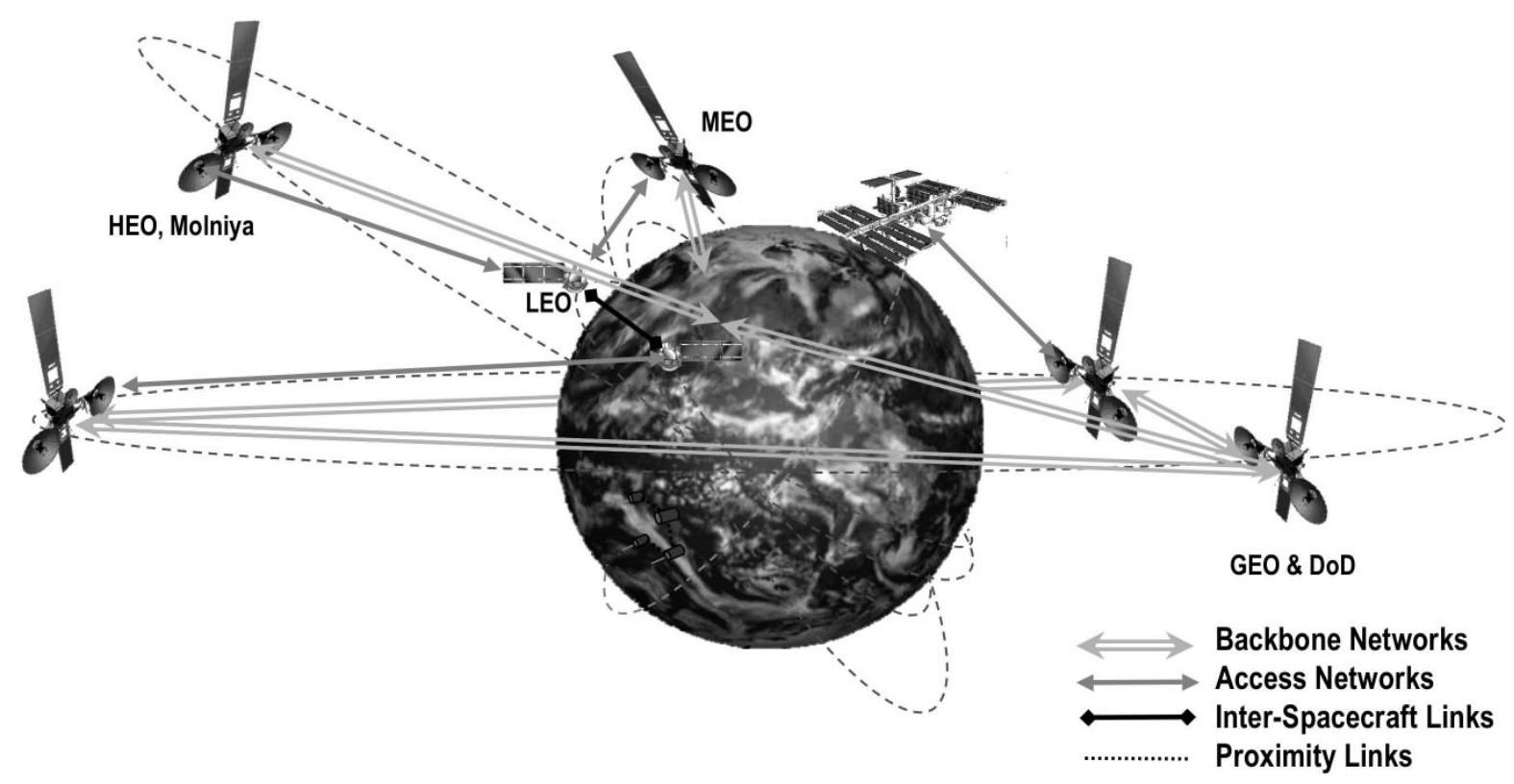

FIGURE 4. Earth Vicinity Nodal Groups. 
Element 1 - Communications relay satellite networks in geosynchronous Earth orbit (GEO). This includes use of the present tracking and data relay satellite system (TDRSS) and any new versions that may be implemented in the future by NASA or the DoD.

Option A - Send and receive data using DoD space networks for NASA science observation and human exploration missions

Option B - Send and receive data using an advanced TDRSS-like space network for NASA science observation and human exploration missions

Element 2 - Communications relay satellite networks that may optionally be placed in high orbit (MEO, HEO, Molniya)

Option A - MEO relay networks

Option B - HEO and Molniya orbit relay networks

Element 3 - LEO Earth observer data and command path

Option A - Send data and receive commands using NASA HEO, GEO, or MEO networks

Option B - LEO satellite sends data and receives commands using TCA space networks

Option C - LEO satellite sends data and receives commands directly with ground terminals

Option D - LEO satellite sends data and receives through science satellites configured as a Sensor

Web

Element 4 - LEO Human mission (such as ISS) data paths

Option A - ISS or shuttle send data and receive commands using NASA HEO, GEO, or MEO

networks

Option B - ISS or shuttle sends data and receives commands using TCA space networks

Option C - ISS or shuttle sends data and receives commands directly with ground terminals

An example of the node-to-node links emanating from a GEO relay satellite is shown in Table 3. While the GEO relay satellite is option B of architectural element 1 in the Earth vicinity, it may also be considered for communicating with missions outside the Earth vicinity. The other mission types that may be communicated with are indicated as connection nodes in the table. It is possible for a GEO relay satellite to capture high rate data from distant nodal regions such as Mars or the outer plants, if the needed technologies can be developed. Tables like Table 2 exist for every architectural element option as a way of cataloging the relative difficulty of implementing each option. While the node-to-node link data has been gathered, the evolutionary mission set is still in formulation. Further data gathering will continue while final selection of the options await a new mission plan.

Table 3. Element 1, Option B. Link Table for Sending and Receiving Data Using an Advanced TDRSS Space Network for NASA Science Observation and Human Exploration Missions.

\begin{tabular}{lccl}
\hline Space Network element link to: & $\begin{array}{c}\text { Data Rate } \\
\text { (Mbps) }\end{array}$ & Distance & Capability \\
\hline NASA LEO satellite & 1,200 & $35,000 \mathrm{~km}$ & Demand access to the IP network. \\
NASA LEO satellite low rate & 10 & $35,000 \mathrm{~km}$ & $\begin{array}{l}\text { Multiple access on-demand to move data, emergency, } \\
\text { TT\&C }\end{array}$ \\
Human spacecraft & 1,200 & $35,000 \mathrm{~km}$ & Bidirectional voice video, data access \\
Space network element (crosslink) & 10,000 & $35,000 \mathrm{~km}$ & Bidirectional backbone data \\
Lunar missions & 1,000 & $0.25 \mathrm{Mkm}$ & Bidirectional voice, HDTV, data \\
Earth-Sun L1, L2 & 300 & $1.5 \mathrm{Mkm}$ & Backbone and Science data \\
Mars missions & 100 & $2.5 \mathrm{AU}$ & Bidirectional voice, HDTV, data \\
Jupiter missions & 16 & $6.2 \mathrm{AU}$ & Science files \\
Saturn missions & 5 & $10.5 \mathrm{AU}$ & Science files \\
Uranus missions & 1.5 & $20.2 \mathrm{AU}$ & Science files \\
Neptune missions & 0.65 & $31.1 \mathrm{AU}$ & Science files \\
\hline
\end{tabular}




\section{Moon Vicinity Communication Nodes}

The Moon vicinity communications infrastructure for robotic and human missions diagrammed in Figure 5 includes Earth-Moon halo orbit, lunar orbit and lunar surface relay satellites that may be implemented.

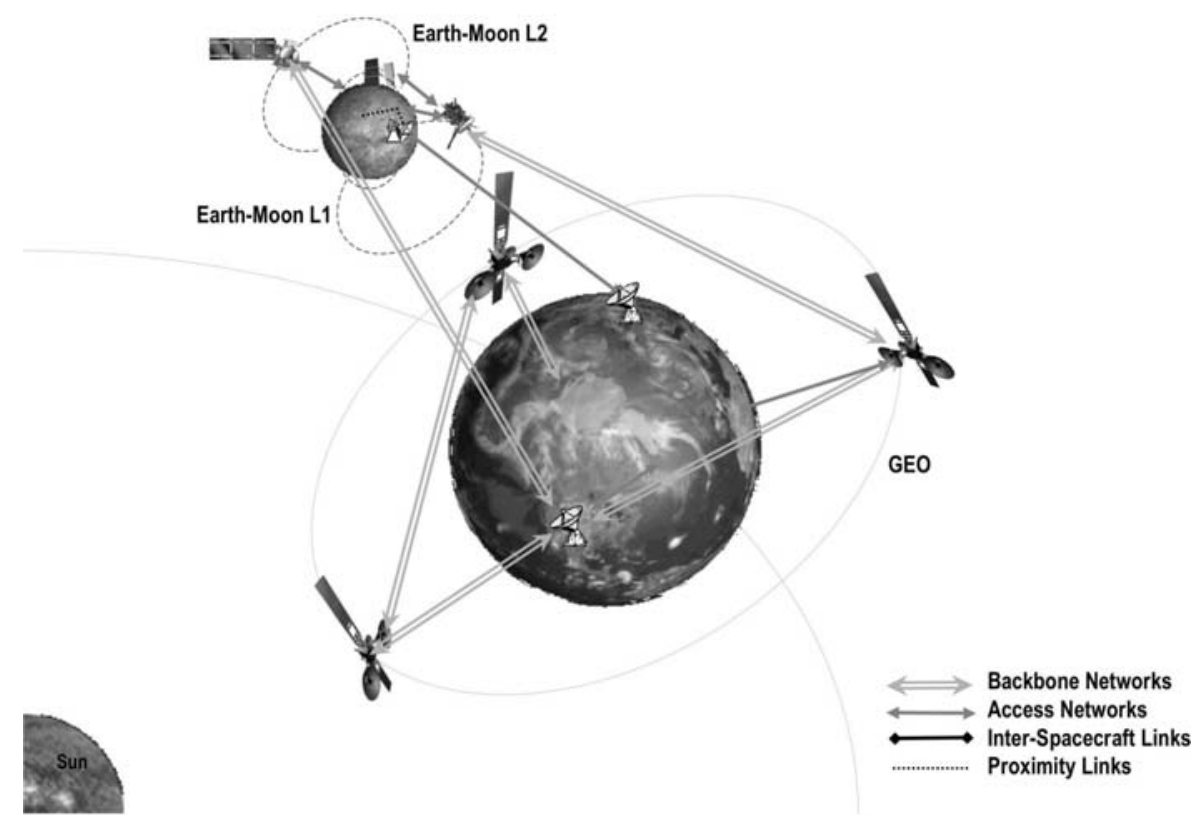

FIGURE 5. Moon Vicinity Nodal Group.

The architectural elements and their options for the Moon vicinity communications infrastructure are listed below. As in the Earth vicinity architectural elements described above, an example of the characterization of one of the option's set of node-to-node links is given below.

Element 1 - Large Satellite Medium Moon Orbit (LSMMO) communications relay satellites

Option A - LSMMO relay spacecraft constellation

Element 2 - Earth-Moon Lagrange orbit communications relay satellites

Option A - Double halo orbit configuration - Earth-Moon L1 relay aboard lunar gateway station

Option B - Double halo orbit configuration - Separate Earth-Moon L1 spacecraft

Option C - Double halo orbit configuration - Earth-Moon L2 relay spacecraft

Element 3 - Small Satellite Low Moon Orbit (SSLMO) communications relay satellites

Option A - Small Satellite, Low Moon Orbit (SSLMO) relay spacecraft constellation

Option B - Small Satellite, Low Moon Orbit (SSLMO) Lunar surface terminal relays

Element 4 - Moon surface communications

Option A - Human lunar outpost sends and receives voice, video, and data using direct to Earth links

Option B - Lunar outpost wireless local area network (WLAN)

An example of the Moon vicinity nodal group is architectural element 1, option A, which is a constellation of 8 Large Satellite in Medium Moon Orbit (LSMMO) communications relays. The satellites are in two orbital planes that are 90 degrees out of phase, one polar and one equatorial orbit at 
$>2000 \mathrm{~km}$ altitude. Three of the satellites in each plane are active, while one is a spare. The node-to-node links for this element are identified in Table 4. The LSMMO configuration provides nearly 24/7 coverage to missions anywhere on the Moon's surface. Data from missions on the far side of the moon are relayed around the constellation and then sent to the Earth. Data from lunar surface to lunar surface are also routed around the constellation. Satellites in this constellation have the ability to communicate with each other, the lunar surface, and with the Earth.

TABLE 4. Element 1, Option A. Link Table for LSMMO Relay Spacecraft Constellation.

\begin{tabular}{lccl}
\hline LSMMO relay spacecraft Link to: & $\begin{array}{c}\text { Data Rate } \\
\text { (Mbps) }\end{array}$ & $\begin{array}{c}\text { Distance } \\
(\mathbf{k m})\end{array}$ & Capability \\
\hline Earth ground & $>300$ & 384,000 & High rate backbone data movement \\
Earth orbit relay & 1,000 & 384,000 & High rate backbone data movement \\
LSMMO relay spacecraft (crosslink) & 1,000 & 6,500 & High rate backbone data movement \\
Moon low rate & 10 & 2,700 & Emergency, TT\&C \\
Moon science orbiter & 100 & 2,700 & Science files \\
Moon human outpost & 1,000 & 2,700 & Bidirectional voice, HDTV, data \\
\hline
\end{tabular}

\section{Nodes for Communications in the Earth-Sun Lagrange Vicinities}

The Earth-Sun Lagrange vicinity communications infrastructure for robotic and human missions is diagrammed in Figure 6 and includes Earth-Sun halo orbit communication relay spacecraft and science spacecraft that may be placed at the Lagrange points.

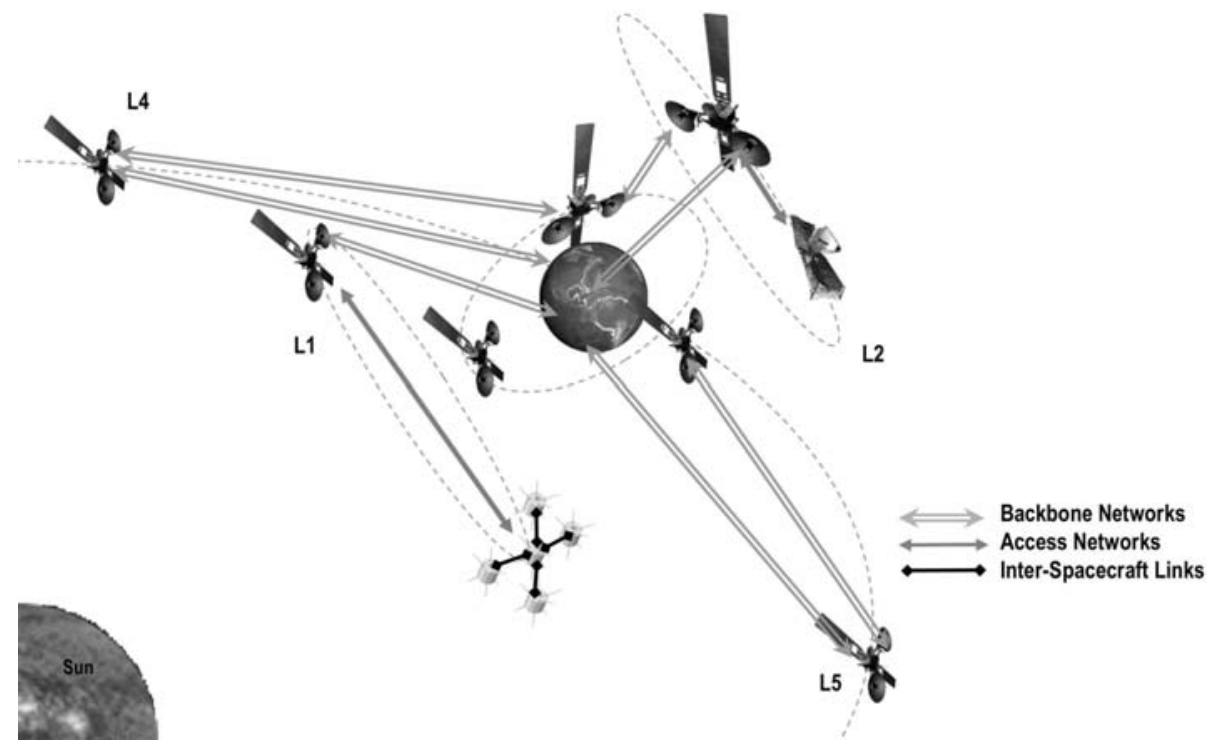

FIGURE 6. Earth-Sun Lagrange Vicinity Nodal Groups.

Element 1 - Earth-Sun L1, L2, L3, L4 communication links

Option A - Earth-Sun L1, L2 relay spacecraft

Option B - Earth-Sun L3, L4 relay spacecraft

Option C - Earth-Sun L1, L2, L3, L4 science spacecraft to Earth-Sun L1, L2, L3, L4 relay spacecraft

Option D - Earth-Sun L1, L2 science spacecraft to Earth direct or Earth relay

Option E - Earth-Sun L4, L5 science spacecraft to Earth direct or Earth relay 
An example of the Earth-Sun Lagrange vicinity nodal groups is architectural element 1, option A, which are communications relay spacecraft placed in halo orbits about L1 and/or L2. These Lagrange halo orbit locations are relatively easy to reach and require very small amounts of propulsive resources to maintain position. The benign gravitational variations at these locations make it easier to meet the extreme pointing accuracy required for very high data rate, fine pointing microwave or laser communication systems. The node-to-node links for these elements are identified in Table 5 . The relays can be used to provide access to the very high data rate Earth space and ground backbones for other spacecraft in local halo orbits as well as provide high rate paths for nodes in other far away regions.

TABLE 5. Element 1, Option A. Link Table for Communication Relays at Earth-Sun L1, and/or L2.

\begin{tabular}{lccl}
\hline L1, L2 Relay Link to: & Data Rate (Mbps) & Distance & Capability \\
\hline Earth ground & $>100$ & $1.5 \mathrm{e} 6 \mathrm{~km}$ & High rate backbone data movement \\
Earth orbit relay & 300 & $1.5 \mathrm{e} 6 \mathrm{~km}$ & High rate backbone data movement \\
Science spacecraft in halo orbit & 300 & $1 \mathrm{e} 6 \mathrm{~km}$ & Access to backbone for science files \\
Mars relays, high rate & 100 & $2.5 \mathrm{AU}$ & High rate backbone data movement \\
Mars low rate & 1 & $2.5 \mathrm{AU}$ & Emergency, TT\&C \\
Mars science S/C & 10 & $2.5 \mathrm{AU}$ & Science files \\
Mars human outpost & 100 & $2.5 \mathrm{AU}$ & Bidirectional voice, HDTV, data \\
Jupiter high rate & 16 & $6.2 \mathrm{AU}$ & Science files \\
Saturn & 5 & $10.5 \mathrm{AU}$ & Science files \\
Uranus & 1.5 & $20.2 \mathrm{AU}$ & Science files \\
Neptune & 0.65 & $31.1 \mathrm{AU}$ & Science files \\
\hline
\end{tabular}

\section{Mars Vicinity Communication Nodes}

The Mars vicinity communications infrastructure for robotic and human missions is diagrammed in Figure 7 along with the deep space communications and includes Mars communication relay satellites, science spacecraft, atmospheric craft, surface rovers, landers, sensor, and human outposts that may be implemented at Mars.

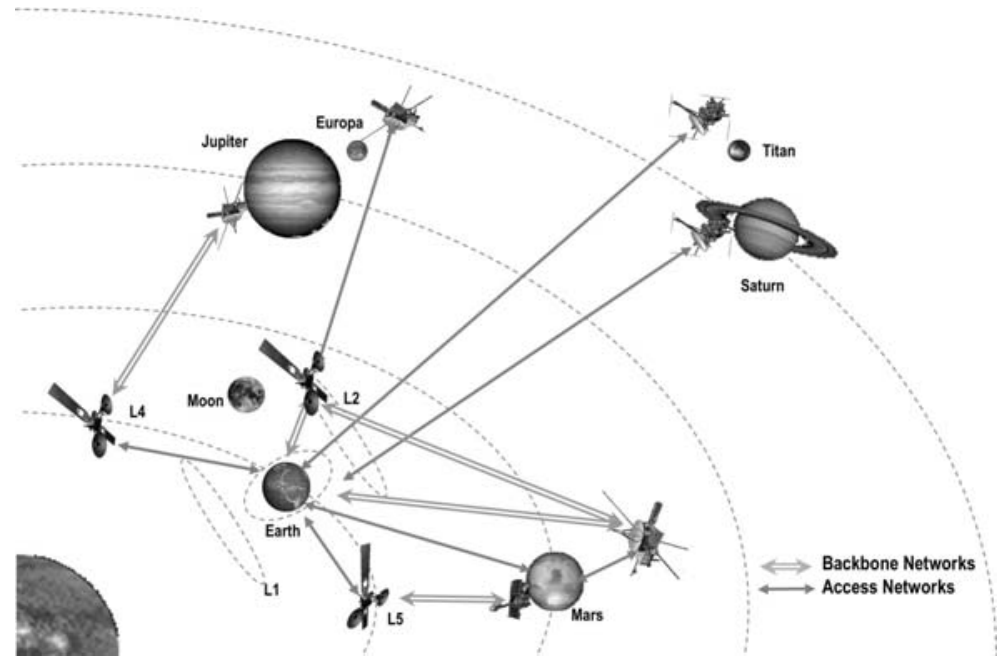

FIGURE 7. Mars Vicinity and Deep Space Nodal Groups. 
Element 1 - A relay satellite network for Mars that might optionally be placed in Mars Synchronous Orbit (MSO), Mars High Orbit (MHO), and/or Mars Low Orbit (MLO)

Option A - MSO communications relay satellite

Option B - HMO communications relay satellite network

Option C - LMO science satellite with add-on relay network function

Element 2 - Networks for Mars orbit, air, and surface robotic missions

Option A - Send data and receive commands using MSO networks

Option B - Send data and receive commands using MHO networks

Option C - Send data and receive commands using MLO networks

Option D - Send data and receive commands using direct to Earth links

Element 2 - Mars Human Outpost communication networks

Option A - Human outpost sends and receives voice, video, and data using MSO networks

Option B - Human outpost sends and receives voice, video, and data using MHO networks

Option C - Human outpost sends and receives voice, video, and data using MLO networks

Option D - Human outpost sends and receives voice, video, and data using direct to Earth links

Option E - Mars outpost wireless local area network (WLAN)

An example of the Mars vicinity nodal groups is architectural element 1, option A, which is one or more communications relay spacecraft placed in Mars synchronous orbit (MSO). A relay satellite placed in MSO can provide 24/7 coverage of one side of Mars. Three MSO satellites would be needed for full Mars coverage. The fixed position above the Mars surface makes it easier for surface entities to find and finely focus communication beams at the communication satellite for very high data rate, fine pointing microwave or laser communications. The node-to-node links for this element are identified in Table 6.

TABLE 6. Element 1, Option A. Link Table for Relays in MSO.

\begin{tabular}{lccl}
\hline MSO Relay Link to: & $\begin{array}{c}\text { Data Rate } \\
\text { (Mbps) }\end{array}$ & Distance & Capability \\
\hline Earth ground & $>1$ & $2.5 \mathrm{AU}$ & Emergency, TT\&C \\
Earth L1, L2, L4, L5, GEO orbit relay & $>100$ & $2.5 \mathrm{AU}$ & Bidirectional Backbone data \\
Mars low rate & 1 & $10,000 \mathrm{~km}$ & Emergency, TT\&C \\
Mars science orbiters & 100 & $10,000 \mathrm{~km}$ & Multiple science S/C files \\
Mars surface robots & 10 & $10,000 \mathrm{~km}$ & Multiple science S/C files \\
Mars human outpost & 100 & $10,000 \mathrm{~km}$ & Bidirectional voice, HDTV, data \\
\hline
\end{tabular}

\section{Deep Space Communication Nodes}

The deep space communications infrastructure for robotic and human missions is diagrammed in Figure 7 along with the Mars vicinity communications and includes communication relay spacecraft in Jupiter-Sun Lagrange halo orbits, science spacecraft, atmospheric craft, surface rovers, landers, and sensors that may be implemented at Jupiter, Saturn, Uranus, Neptune, their moons or other objects in the vicinity. The infrastructure also includes the possible support of human missions to the Jupiter moons. 
TABLE 7. Link Table for Science Spacecraft in Jupiter Orbit.

\begin{tabular}{|c|c|c|c|}
\hline $\begin{array}{l}\text { Science Spacecraft in Planet Orbit } \\
\text { Link to: }\end{array}$ & $\begin{array}{c}\text { Data Rate } \\
\text { (Mbps) }\end{array}$ & Distance & Capability \\
\hline \multicolumn{4}{|c|}{ Science spacecraft in Jupiter orbit link to: } \\
\hline Earth ground & 1 & $6.2 \mathrm{AU}$ & Emergency, TT\&C \\
\hline Earth L1, L2, L4, L5, GEO orbit relay & 16 & $6.2 \mathrm{AU}$ & Bidirectional Backbone data \\
\hline Jupiter moon surface robots & $1-10$ & $400-10,000 \mathrm{~km}$ & Multiple science S/C files, emergency, TT\&C \\
\hline \multicolumn{4}{|l|}{ Science spacecraft in Saturn orbit link to: } \\
\hline Earth ground & 0.1 & 10.5 AU & Emergency, TT\&C \\
\hline Earth L1, L2, L4, L5, GEO orbit relay & 5 & $10.5 \mathrm{AU}$ & Science files \\
\hline Saturn moon surface robots & $1-10$ & $400 \mathrm{~km}$ & Multiple science S/C files, emergency, TT\&C \\
\hline \multicolumn{4}{|l|}{ Science spacecraft in Uranus orbit link to: } \\
\hline Earth ground & 0.02 & $20.2 \mathrm{AU}$ & Emergency, TT\&C \\
\hline Earth L1, L2, L4, L5, GEO orbit relay & 1.5 & $20.2 \mathrm{AU}$ & Science files \\
\hline Uranus moon surface robots & $1-10$ & $400 \mathrm{~km}$ & Multiple science S/C files, emergency, TT\&C \\
\hline \multicolumn{4}{|c|}{ Science spacecraft in Neptune orbit link to: } \\
\hline Earth ground & 0.01 & $31.1 \mathrm{AU}$ & Emergency, TT\&C \\
\hline Earth L1, L2, L4, L5, GEO orbit relay & 16 & $31.1 \mathrm{AU}$ & Science files \\
\hline Neptune moon surface robots & $1-10$ & $400 \mathrm{~km}$ & Multiple science S/C files, emergency, TT\&C \\
\hline
\end{tabular}

An example of the deep space nodal groups is architectural element 1, option A. This element option is the communication package on-board each science spacecraft that visits an outer planet object. These communication packages can only cover an area on a moon or the planet that is within a cone of visibility directly below the spacecraft. Pointing, acquisition, and tracking for capturing high data rate communications from a vehicle in or on an outer planet object and then relaying it to Earth or to Earth relay assets is dynamic, complex, and difficult due to the dynamic orbital motion of the spacecraft and the divergent (Earth, vehicle) pointing requirements. The node-to-node links for this element are identified in Table 7.

\section{Conclusions}

Space communications architectures concept, design and analysis will play a key role in the development and deployment of NASA's future exploration and science missions to realize maximum return on investment. Once the mission is deployed the communication link to the user needs to provide maximum information delivery and flexibility to alter the outcome in a timely fashion, Furthermore, in human and robotic missions it needs to offer maximum reliability with robust two way links for software uploads and virtual interactions. These requirements can only be met with architecture design and early technology developments.

In this paper, we have made an attempt to define and model a space communication architecture that can meet the challenging requirements for evolutionary missions. The systematic identification of the communications architectural elements and the optional ways they can be implemented serves as a valuable tool for indicating to the mission planner and scientist the possible communication capabilities that can be realized by the alternate configurations. It serves well for constructing strawman architectures for evaluation of which options have the highest payback potential. Extensive system cost and risk analysis and trades will be the next logical step to refine the architecture for implementation.

\section{References}

1. Shames, P, “Architectural Models of Space Networking,” (2003), http://scp.grc.nasa.gov/siw/presentations/Session_B/B_06_ Shames.pdf Accessed October 14, 2003.

2. Bhasin, K., and Hayden, J.L., Int. J. Satell. Commun. 2002; 20, 311-332. 
Public reporting burden for this collection of information is estimated to average 1 hour per response, including the time for reviewing instructions, searching existing data sources, gathering and maintaining the data needed, and completing and reviewing the collection of information. Send comments regarding this burden estimate or any other aspect of this collection of information, including suggestions for reducing this burden, to Washington Headquarters Services, Directorate for Information Operations and Reports, 1215 Jefferson Davis Highway, Suite 1204, Arlington, VA 22202-4302, and to the Office of Management and Budget, Paperwork Reduction Project (0704-0188), Washington, DC 20503.

1. AGENCY USE ONLY (Leave blank) 2. REPORT DATE 3. REPORT TYPE AND DATES COVERED

\section{TITLE AND SUBTITLE}

April 2004

Technical Memorandum

Evolutionary Space Communications Architectures for Human/Robotic

Exploration and Science Missions 5. FUNDING NUMBERS

6. AUTHOR(S)

Kul Bhasin and Jeffrey L. Hayden

WBS-22-704-302-20-Z

National Aeronautics and Space Administration

John H. Glenn Research Center at Lewis Field

Cleveland, Ohio 44135-3191

8. PERFORMING ORGANIZATION REPORT NUMBER

E-14550

\section{SPONSORING/MONITORING AGENCY NAME(S) AND ADDRESS(ES)}

10. SPONSORING/MONITORING AGENCY REPORT NUMBER

National Aeronautics and Space Administration

Washington, DC 20546-0001

NASA TM-2004-213074

\section{SUPPLEMENTARY NOTES}

Prepared for the Space Technology and Applications International Forum (STAIF-2004) sponsored by the American Institute of Physics, Albuquerque, New Mexico, February 8-12, 2004. Kul Bhasin, NASA Glenn Research Center; and Jeffrey L. Hayden, PresciPoint Solutions, L.L.C., Littleton, Colorado 80123. Responsible person, Kul Bhasin, organization code 6100, 216-433-3676.

12a. DISTRIBUTION/AVAILABILITY STATEMENT

12b. DISTRIBUTION CODE

Unclassified - Unlimited

Subject Category: 32

Distribution: Nonstandard

Available electronically at http://gltrs.grc.nasa.gov

This publication is available from the NASA Center for AeroSpace Information, 301-621-0390.

\section{ABSTRACT (Maximum 200 words)}

NASA enterprises have growing needs for an advanced, integrated, communications infrastructure that will satisfy the capabilities needed for multiple human, robotic and scientific missions beyond 2015. Furthermore, the reliable, multipoint infrastructure is required to provide continuous, maximum coverage of areas of concentrated activities, such as around Earth and in the vicinity of the Moon or Mars, with access made available on demand of the human or robotic user. As a first step, the definitions of NASA's future space communications and networking architectures are underway. Architectures that describe the communications and networking needed between the nodal regions consisting of Earth, Moon, Lagrange points, Mars, and the places of interest within the inner and outer solar system have been laid out. These architectures will need the modular flexibility that must be included in the communication and networking technologies to enable the infrastructure to grow in capability with time and to transform from supporting robotic missions in the solar system to supporting human ventures to Mars, Jupiter, Jupiter's moons, and beyond. The protocol-based networking capability seamlessly connects the backbone, access, inter-spacecraft and proximity network elements of the architectures employed in the infrastructure. In this paper, we present the summary of NASA's near and long term needs and capability requirements that were gathered by participative methods. We describe an integrated architecture concept and model that will enable communications for evolutionary robotic and human science missions. We then define the communication nodes, their requirements, and various options to connect them.

14. SUBJECT TERMS

Space communications; Interplanetary communication; Lunar communication 15. NUMBER OF PAGES 19

17. SECURITY CLASSIFICATION OF REPORT

Unclassified

18. SECURITY CLASSIFICATION
OF THIS PAGE
Unclassified

Unclassified

\section{SECURITY CLASSIFICATION OF ABSTRACT \\ Unclassified}



